\title{
Amphipathic Polymers: Tools To Fold Integral Membrane Proteins to Their Active Form ${ }^{\dagger}$
}

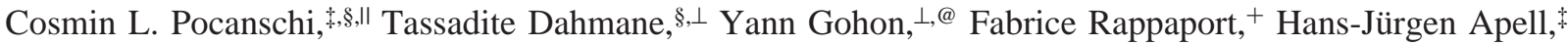

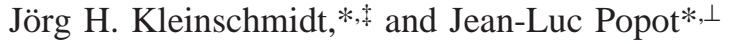 \\ Fachbereich Biologie, Fach M 694, Universität Konstanz, D-78457 Konstanz, Germany, and UMR 7099, CNRS, and \\ Université Paris-7, and UMR 7141, CNRS, and Université Paris-6, Institut de Biologie Physico-Chimique, \\ CNRS FRC 550, 13 rue Pierre et Marie Curie, F-75005 Paris, France
}

Received August 16, 2006; Revised Manuscript Received September 27, 2006

\begin{abstract}
Among the major obstacles to pharmacological and structural studies of integral membrane proteins (MPs) are their natural scarcity and the difficulty in overproducing them in their native form. MPs can be overexpressed in the non-native state as inclusion bodies, but inducing them to achieve their functional three-dimensional structure has proven to be a major challenge. We describe here the use of an amphipathic polymer, amphipol A8-35, as a novel environment that allows both $\beta$-barrel and $\alpha$-helical MPs to fold to their native state, in the absence of detergents or lipids. Amphipols, which are extremely mild surfactants, appear to favor the formation of native intramolecular protein-protein interactions over intermolecular or protein-surfactant ones. The feasibility of the approach is demonstrated using as models OmpA and FomA, two outer membrane proteins from the eubacteria Escherichia coli and Fusobacterium nucleatum, respectively, and bacteriorhodopsin, a light-driven proton pump from the plasma membrane of the archaebacterium Halobacterium salinarium.
\end{abstract}

Pharmacological and structural studies of integral membrane proteins (MPs), ${ }^{1}$ which play fundamental roles in cell physiology, are hampered by their scarcity. Overexpression is generally difficult. Schematically, heterologously expressed MPs targeted to the plasma membrane tend to kill their host, limiting yields, while those directed to nontoxic inclusion bodies are recovered in a non-native state and most often are impossible to fold with acceptable yields $(1,2)$. Refolding MPs until now has relied on transfer from a denaturant solution to lipids, detergents, or mixtures thereof (for reviews,

† This work was supported by Grants KL 1024/4-1 and 4-2 from the Deutsche Forschungsgemeinschaft to J.H.K. and by the Centre National de la Recherche Scientifique, Université Paris-7, Human Frontier Science Program Organization Grant RG00223/2000-M and E.U. Specific Targeted Research Project "Innovative tools for membrane protein structural proteomics" to J.-L.P. T.D. was the recipient of a fellowship from the Ministère de l'Enseignement Supérieur et de la Recherche.

* To whom correspondence should be addressed. E-mail: joerg.helmut.kleinschmidt@uni-konstanz.de and jean-luc.popot@ibpc.fr.

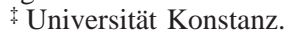

$\S$ These authors contributed equally to this work.

" Present address: Department of Biochemistry, Medical Sciences Building, University of Toronto, Toronto, Ontario, Canada M5S 1A8.

${ }^{\perp}$ UMR 7099, CNRS and Université Paris-7.

@ Present address: UMR INRA-INAPG 206, Laboratoire de Chimie Biologique, F-78850 Thiverval-Grignon, France.

+ UMR 7141, CNRS and Université Paris-6.

${ }^{1}$ Abbreviations: A8-35, a specific form of amphipol (Figure 1A); APol, amphipol; BO, bacterioopsin; BR, bacteriorhodopsin; CD, circular dichroism; $\mathrm{dBO}$ or delipidated $\mathrm{BO}, \mathrm{BO}$ separated from $\mathrm{PM}$ lipids by solubilization in organic solvents; diPhPC, diphytanoylphosphatidylcholine; KDS and SDS, potassium and sodium dodecyl sulfate, respectively; MP, membrane protein; LDAO, $N$-lauryl- $N, N$-dimethylamine $N$-oxide; OTG, octyl thioglucoside; PAGE, polyacrylamide gel electrophoresis; PM, purple membrane; 3D, three-dimensional; UV, ultraviolet. see, for example, refs $3-6$ ). We report here on the use of a new refolding medium comprised of synthetic, polymeric surfactants called amphipols (APols).

APols are short amphipathic polymers comprised of a hydrophilic backbone grafted with alkyl chains (7), which provide solubilized MPs with an environment milder and less denaturing than that provided by detergent solutions (reviewed in ref 8 ). The reasons why APols stabilize MPs are multifarious (refs 8 and 9 and unpublished data), but they most certainly include their limited efficiency at disrupting protein-protein and protein-lipid interactions. On the assumption that the dissociating character of detergents is one of the major hindrances in renaturation attempts, we have explored the use of APols to refold MPs. The polymer used for this study, A8-35, is a derivative of polyacrylic acid (ref 7 and Figure 1A). Its synthesis and solution properties have been studied in detail $(10,11)$, and its mildness toward MPs has been documented in several studies (e.g., refs 7, 9, and 12 ; reviewed in ref 8 ). A8-35 is by far the best characterized APol to date and seems to have interesting prospects for membrane protein biochemical and biophysical investigations (see, for example, refs 8 and 13-15). APol-mediated refolding was tested on three MPs from the two main structural classes, namely, two $\beta$-barrel proteins from eubacterial outer membranes, OmpA from Escherichia coli and FomA from Fusobacterium nucleatum, and the $\alpha$-helical MP bacteriorhodopsin from the plasma membrane of the archaebacterium Halobacterium salinarium.

\section{EXPERIMENTAL PROCEDURES}

Chemicals. SDS was from Bio-Rad. Formic acid (88\%, HPLC-grade) was from Fluka. Ethanol (HPLC-grade) was 


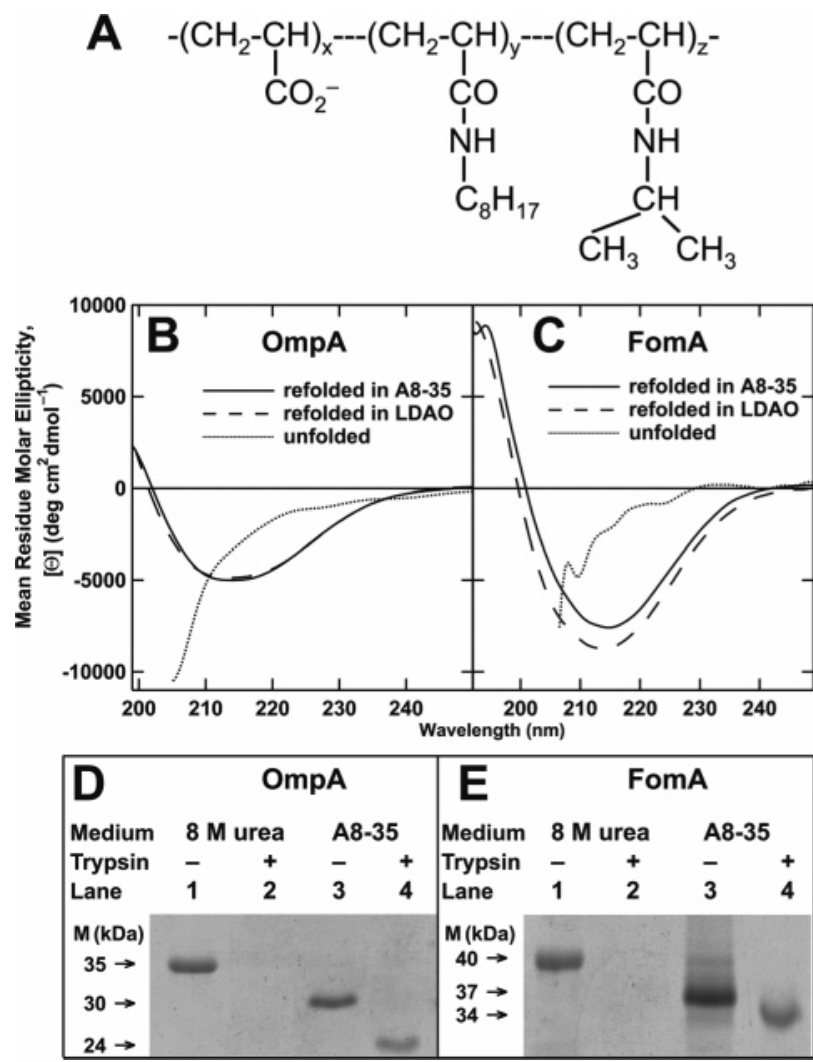

FIGURE 1: (A) Chemical structure of APol A8-35, where $\langle x\rangle \approx$ $35 \%,\langle y\rangle \approx 25 \%,\langle z\rangle \approx 40 \%$, and $\langle\mathrm{MW}\rangle=9-10 \mathrm{kDa}(7)$. (B and C) CD spectra of OmpA (B) and FomA (C) recorded before and after refolding in A8-35. Spectra of OmpA and FomA folded in LDAO, which are similar to those of the native proteins $(32,35)$, are shown for comparison. (D and E) Migration of OmpA (D) and FomA (E) on a SDS-PAGE gel, indicating folding and protection against trypsin digestion by APol A8-35: lane 1, denatured MP in $8 \mathrm{M}$ urea; lane 2, same incubated for $2 \mathrm{~h}$ with trypsin; lane 3 , refolded MP in A8-35; and lane 4, same incubated for $2 \mathrm{~h}$ with trypsin.

from Carlo Erba Réactifs/Solvants, Documentation, Synthèses. Sephadex LH-60 and Superose 12/HR were from Pharmacia. Sodium azide, soybean trypsin inhibitor (type I-S), and all-trans-retinal were from Sigma. Diphytanoylglycerophosphocholine (diPhPC) was from Avanti Polar Lipids (Alabaster, AL). Bacteriological peptone was from Oxoid. Trisodium citrate, potassium chloride, and magnesium sulfate were from Normapur, and other salts were from Sigma. All aqueous solutions were made in water purified on a Milli-Q system (Millipore). A8-35 (batches FGH15 and FGH20) was synthesized and purified by F. Giusti (UMR 7099) as described in refs 10 and 11 .

Buffers. Borate/ $\mathrm{NaOH}$ buffer consisted of $10 \mathrm{mM}$ borate ( $\mathrm{pH} 10.0)$ and $2 \mathrm{mM}$ EDTA. KCl/Tris buffer consisted of 1 $\mathrm{M} \mathrm{KCl}$ and $10 \mathrm{mM}$ Tris ( $\mathrm{pH}$ 7.2). SDS buffer consisted of $5 \%(\mathrm{w} / \mathrm{v}) \mathrm{SDS}, 0.025 \%(\mathrm{w} / \mathrm{v}) \mathrm{NaN}_{3}$, and $50 \mathrm{mM}$ sodium phosphate ( $\mathrm{pH} 7)$. K buffer consisted of $150 \mathrm{mM} \mathrm{KCl}$, $0.025 \% \mathrm{NaN}_{3}$, and $30 \mathrm{mM}$ potassium phosphate ( $\left.\mathrm{pH} 7\right) . \mathrm{Na}$ buffer consisted of $100 \mathrm{mM} \mathrm{NaCl}, 0.025 \% \mathrm{NaN}_{3}$, and 20 $\mathrm{mM}$ potassium phosphate $(\mathrm{pH} 7)$.

Isolation of OmpA and FomA. OmpA was isolated from E. coli as described previously (16) and stored in the unfolded form in borate/ $\mathrm{NaOH}$ buffer containing $8 \mathrm{M}$ urea. E. coli $\mathrm{B}$ strain PC2889 harboring the pET-10953 plasmid for expression of FomA was a generous gift from H. Jensen (University of Bergen, Bergen, Norway). FomA was isolated as previously described (17). Inclusion bodies of FomA were solubilized in borate/ $\mathrm{NaOH}$ buffer containing $10 \mathrm{M}$ urea. The absence of lipids in the preparations (cf. ref 16) was routinely checked by phosphate determination.

Preparation of SDS-Solubilized Purple Membrane. H. salinarium (strain S9, a gift of G. Zaccaï, IBS, Grenoble, France) was grown on bacteriological peptone $(12 \mathrm{~g} / \mathrm{L})$ and purple membrane (PM) isolated as described previously (18). BR was denatured by incubating PM (1 g/L) in SDS buffer for $2 \mathrm{~h}$ at room temperature in the dark. The material was totally solubilized (no pellet after centrifugation for $10 \mathrm{~min}$ at $80000 \mathrm{~g}$ ). Visible spectra indicated the complete absence of native BR (absence of an absorbance peak around 550 nm; see Figure 3A).

Preparation of SDS-Solubilized Delipidated Bacterioopsin $(d B O)$. Lyophilized PM was solubilized at room temperature in $88 \%$ formic acid, as described in refs 19 and 20 for bleached PM. Addition of formic acid entailed immediate bleaching of BR, as reflected by the color of the sample turning from purple to yellow. After $5 \mathrm{~min}$, the solution was supplemented with ethanol to yield a 30/70 (v/v) formic acid/ ethanol mixture. The final protein concentration was typically $4 \mathrm{~g} / \mathrm{L}$. The separation of $\mathrm{dBO}$ from lipids and retinal was performed by size exclusion chromatography in formic acid and ethanol on a 200-mL Sephadex LH-60 column $(19,20)$. The absence of lipids in dBO fractions ( $<2 \%$ of the lipid/ $\mathrm{BR}$ ratio in $\mathrm{PM}$ ) was established by thin layer chromatography using $10 \mathrm{~cm} \times 12 \mathrm{~cm}$ silica plates and a solvent mixture of chloroform, $90 \%$ acetic acid, and methanol (65/ $35 / 4$, by volume) (21). The transfer of $\mathrm{dBO}$ from formic acid and ethanol to SDS was carried out by dialysis as described in ref 20. The final concentration of $\mathrm{dBO}$ in SDS buffer was $\sim 0.25 \mathrm{~g} / \mathrm{L}$, taking $\epsilon_{280}$ for BO to be $65 \mathrm{mM}^{-1} \mathrm{~cm}^{-1}$ (22). Before renaturation, $\mathrm{dBO}$ solutions in SDS buffer were supplemented with retinal in a $1.3 / 1 \mathrm{retinal} / \mathrm{dBO}$ molar ratio, taking $\epsilon_{382}$ for retinal to be $42.8 \mathrm{mM}^{-1} \mathrm{~cm}^{-1}$ (23).

Refolding of Outer Membrane Proteins. For SDS-PAGE analyses, refolding of OmpA was initiated by rapid 20-fold dilution of denatured OmpA in borate/ $\mathrm{NaOH}$ buffer containing $8 \mathrm{M}$ urea into urea-free buffer containing $8 \mathrm{~g}$ of $\mathrm{A} 8$ 35/g of OmpA (i.e., an A8-35/OmpA molar ratio of $~ 30$ ). The final concentrations were $15 \mu \mathrm{M}$ and $4.2 \mathrm{~g} / \mathrm{L}$ for OmpA and A8-35, respectively. Similarly, refolding of FomA was performed by diluting a solution in $10 \mathrm{M}$ urea into 19 volumes of borate buffer containing $8.5 \mathrm{~g}$ of $\mathrm{A} 8-35 / \mathrm{g}$ of FomA (i.e., an $\sim 35 / 1$ molar ratio). The final concentrations were $11 \mu \mathrm{M}$ and $3.8 \mathrm{~g} / \mathrm{L}$ for FomA and A8-35, respectively. Samples were incubated at $40{ }^{\circ} \mathrm{C}$ for $24 \mathrm{~h}$.

For CD spectroscopy, refolding was initiated by rapid 20fold (OmpA) or 10-fold (FomA) dilution of the urea-unfolded proteins into urea-free buffer at an A8-35/protein ratio of 2/1 (w/w). Alternatively, OmpA and FomA were refolded in LDAO by diluting the urea-unfolded proteins 20 -fold into urea-free buffer containing $48 \mathrm{mM}$ LDAO (OmpA) or 93 $\mathrm{mM}$ LDAO (FomA) (6.7 or $4.3 \mathrm{~g}$ of $\mathrm{LDAO} / \mathrm{g}$ of $\mathrm{MP}$, respectively). The final concentration of refolded OmpA in A8-35 or LDAO was $46 \mu \mathrm{M}$. The final concentration of refolded FomA was $22 \mu \mathrm{M}$ in A8-35 and $111 \mu \mathrm{M}$ in LDAO. All samples were subsequently incubated for $24 \mathrm{~h}$ at $40{ }^{\circ} \mathrm{C}$. Prior to CD spectroscopy, refolded samples of OmpA and FomA were dialyzed at $10{ }^{\circ} \mathrm{C}$ for $12 \mathrm{~h}$ against $2 \mathrm{~L}$ of borate/ 
$\mathrm{NaOH}$ buffer to remove urea, which causes high noise levels below $205 \mathrm{~nm}$. The dialysis buffer was exchanged three times.

Protease Digestion. Protection of the transmembrane domains of APol-refolded OmpA and FomA against proteolysis was tested by trypsin digestion as described previously (16). Samples at $0.4 \mathrm{~g} / \mathrm{L}$ were incubated with trypsin at $0.04 \mathrm{~g} / \mathrm{L}$ at $37{ }^{\circ} \mathrm{C}$ for $2 \mathrm{~h}$. Digestion was stopped by addition of $0.04 \mathrm{~g} / \mathrm{L}$ soybean trypsin inhibitor. For SDSPAGE, an equal volume of $0.125 \mathrm{M}$ Tris buffer ( $\mathrm{pH}$ 6.8) containing 4\% SDS, $20 \%$ glycerol, 10\% 2-mercaptoethanol, and $0.01 \%$ bromophenol blue was added and $4 \mu \mathrm{g}$ of MP was applied to each lane of a $12 \%$ polyacrylamide gel.

Reconstitution of Refolded Outer Membrane Proteins into Black Lipid Films and Single-Channel Conductance Experiments. Bilayers were formed from a $1 \%$ solution of $d i \mathrm{PhPC}$ in $n$-decane. The lipid solution was painted over a $500 \mu \mathrm{m}$ hole in a Teflon partition separating two $6 \mathrm{~mL}$ compartments filled with $\mathrm{KCl} /$ Tris buffer (24). The compartments were connected to the recording system via two silver electrodes coated with silver chloride, one of which (cis side) was grounded and the other of which (trans side) was connected to a custom-designed trans-impedance amplifier. For reconstitution, MP-A8-35 complexes were simply added to the cis compartment of the Teflon chamber. The films were tested for integrity by checking their reflectance, resistance, and capacitance. A voltage of $100 \mathrm{mV}(\mathrm{OmpA})$ or $10 \mathrm{mV}$ (FomA) was applied $(25,26)$. Current signals were recorded at $22{ }^{\circ} \mathrm{C}$, with a frequency bandwidth of $100 \mathrm{~Hz}$.

Renaturation of Bacteriorhodopsin. BR renaturation was carried out by precipitating dodecyl sulfate as its potassium salt (KDS), as described in ref 20, except for the substitution of A8-35 to the mixture of lipids and taurocholate used in the earlier work. A8-35 was added, at ratios of 2, 5, or $10 \mathrm{~g}$ of A8-35/g of BO, either to SDS-solubilized PM or to dBO in SDS buffer supplemented with retinal at a 1.3/1 retinal/ BO molar ratio. After a $15 \mathrm{~min}$ incubation at room temperature, the solution was supplemented, while being vigorously stirred, with enough $\mathrm{KCl}(4 \mathrm{M})$ to bring the nominal concentration of free $\mathrm{K}^{+}$ions to $150 \mathrm{mM}$ (see ref 20). After $30 \mathrm{~min}$, the KDS precipitate was removed by two 5 -min centrifugations at $6000 \mathrm{rpm}(\sim 6000 \mathrm{~g})$ in a Hettich Mikro $12-24$ centrifuge. The supernatant, initially slightly turbid due to the presence of KDS microcrystallites, was dialyzed twice against 100 volumes of $\mathrm{K}$ buffer for a total of $48 \mathrm{~h}$ at room temperature to remove residual dodecyl sulfate. The final concentration of renatured BR was in the range of $0.2-$ $0.4 \mathrm{~g} / \mathrm{L}$.

Characterization of Renatured BR Samples. The extent of renaturation was estimated by comparing the $A_{280} / A_{554}$ ratio of dark-adapted samples to that of native BR in OTG. Spectra were recorded at room temperature in $100 \mu \mathrm{L}$ quartz cuvettes (Hellma) on a Hewlett-Packard HP-8453 spectrophotometer. Stokes radii were determined by size exclusion chromatography on a Superose 12/HR column equilibrated in K buffer, connected to an Äkta system (Pharmacia). Elution profiles were typically followed at 280,382 , and $554-570 \mathrm{~nm}$. Light adaptation was induced by exposing the sample to white light for 4 min at $4{ }^{\circ} \mathrm{C}$.

Circular Dichroism Spectroscopy and Spectral Analysis. Far-UV CD spectra of $46 \mu \mathrm{M}$ OmpA and 22 or $111 \mu \mathrm{M}$ FomA were recorded on a Jasco $715 \mathrm{CD}$ spectropolarimeter using a a $0.1 \mathrm{~mm}$ cuvette. Twelve scans, from 200 to 250 $\mathrm{nm}$ (from 205 to $250 \mathrm{~nm}$ for unfolded protein in urea) with a response time of $16 \mathrm{~s}$, a bandwidth of $1 \mathrm{~nm}$, and a scan speed of $20 \mathrm{~nm} / \mathrm{min}$, were accumulated and averaged. Background spectra without MP were subtracted for all samples. The recorded CD spectra were normalized to obtain the mean residue molar ellipticity, $[\Theta](\lambda)$, in degrees square centimeters per decimole:

$$
[\Theta](\lambda)=100 \frac{\Theta(\lambda)}{c \cdot n \cdot l}
$$

where $l$ is the path length of the cuvette in centimeters, $\Theta(\lambda)$ is the recorded ellipticity in degrees at wavelength $\lambda$, $c$ is the concentration in moles per liter, and $n$ is the number of amino acid residues, 325 for OmpA and 352 for FomA.

Time-Resolved Absorption Spectroscopy. Absorption changes associated with the photocycle of BR were recorded on a home-built instrument (27). The photocycle was triggered by a $640 \mathrm{~nm}$ pulse with a duration of $5 \mathrm{~ns}$ (FWHM) from a frequency-doubled Nd:Yag laser. Time-resolved absorption changes were followed using monochromatic laser pulses provided by an optical parametric oscillator. The time resolution of the setup is $5 \mathrm{~ns}$. The spectral range that was analyzed extends from 370 to $500 \mathrm{~nm}$.

\section{RESULTS}

Refolding Two $\beta$-Barrel Membrane Proteins, OmpA and FomA. The $171 \mathrm{~N}$-terminal residues of OmpA span the outer membrane of $E$. coli as an eight-stranded $\beta$-barrel (28), while its $154 \mathrm{C}$-terminal residues form a periplasmic domain. OmpA functions as a small ion channel (25) and as a structural protein $(29,30)$. Previous studies have shown that, following transfer from detergent solution to A8-35, (i) the transmembrane domain of OmpA retains its native threedimensional (3D) structure and (ii) the polymer associates noncovalently but very tightly with the hydrophobic surface of the $\beta$-barrel $(15,31)$. FomA, thought to fold into a 14strand transmembrane $\beta$-barrel (32), forms voltage-dependent channels and is likely involved in the binding of fusobacteria and Streptococcus sanguis to the surface of teeth (see ref 33 and references therein). Its interactions with APols have not been studied previously.

When solutions of urea-unfolded OmpA or FomA were diluted 20-fold in urea-free buffer in the presence of A8-35, both proteins remained soluble, instead of aggregating and precipitating as they do in the absence of surfactant (34). Circular dichroism $(\mathrm{CD})$ spectra (Figure 1B,C) were similar to those of native OmpA and FomA in detergent micelles $(32,35)$.

Folded and unfolded forms of OmpA and FomA can be distinguished by sodium dodecyl sulfate-polyacrylamide gel electrophoresis (SDS-PAGE), provided the samples are not heat-denatured. The apparent molecular masses of the unfolded and native forms are 35 and $30 \mathrm{kDa}$ for OmpA (36) and 40 and $37 \mathrm{kDa}$ for FomA (37), respectively. When OmpA and FomA, transferred from urea to A8-35, were analyzed by SDS-PAGE, both proteins migrated as their native form (Figure 1D,E).

The degree of membrane insertion of OmpA and FomA can be determined by protease digestion, their transmembrane domains being protected by the lipid bilayer $(33,36)$. In 

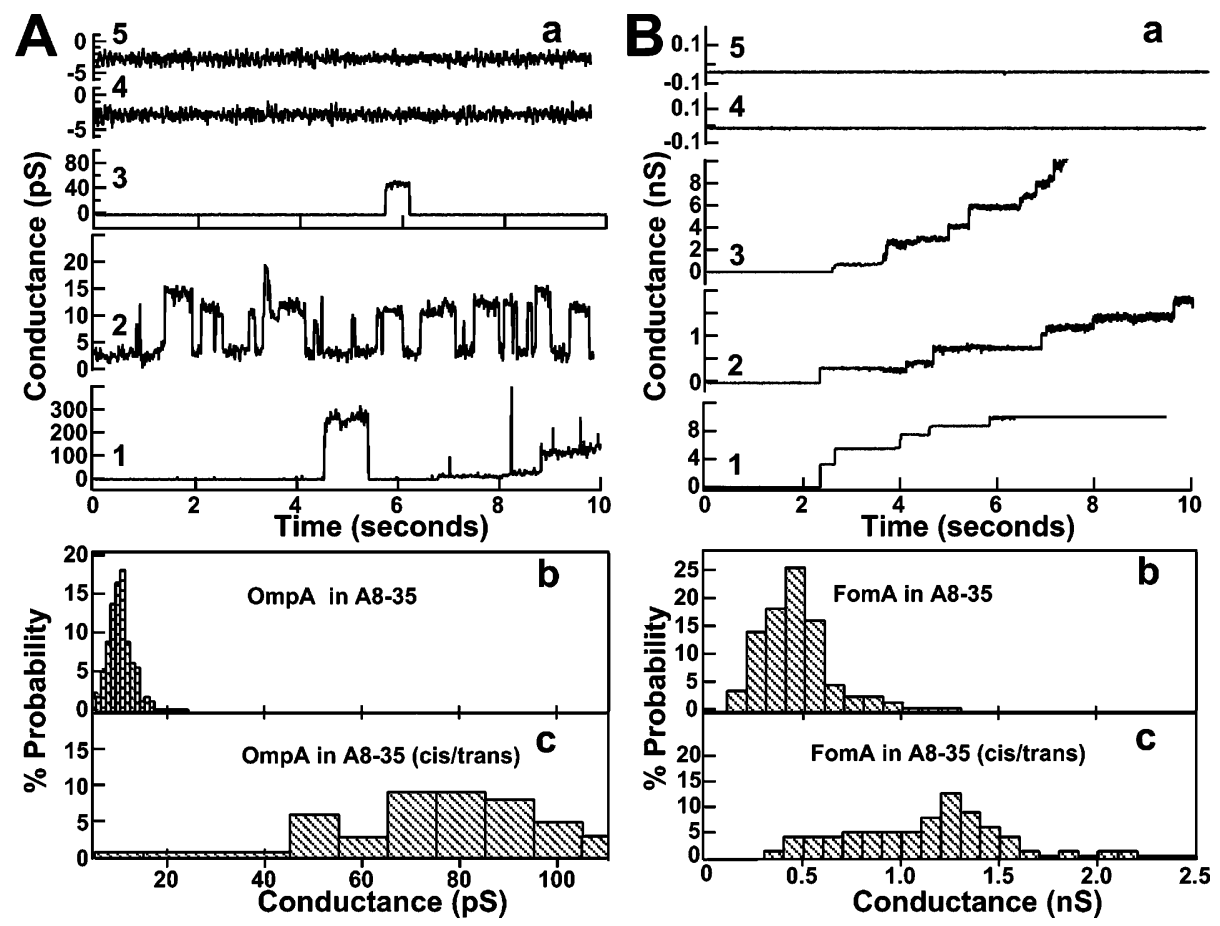

FIGURE 2: Single-channel recordings of refolded OmpA and FomA integrated into black lipid films. (A) OmpA. (a) Large (1) and small (2) conductance states observed when A8-35-refolded OmpA was added to the cis side of a diPhPC film. The conductance of the small state increased when A8-35 was added to the trans side (3). No event was observed in the presence of A8-35 alone, whether added in trans (not shown), in cis (4), or on both sides of the film (not shown), or in that of unfolded OmpA (5). (b and c) Distribution of small conductance states observed after addition of A8-35-OmpA complexes to the cis side in the absence (b) or presence (c) of A8-35 on the trans side. (B) FomA. (a) Channels formed by A8-35-refolded FomA added to the cis side with A8-35 present (1) or absent (2) in the trans compartment and control experiments with LDAO-refolded FomA (3), with A8-35 only (on the cis side) (4) and with denatured FomA (5). (b and c) Distribution of conductance states observed after addition of A8-35-FomA complexes to the cis side without (b) and with (c) A8-35 added to the trans side.

agreement with NMR data (15), A8-35 was found to protect the transmembrane domain of refolded OmpA against proteolysis, leading to the production of a $24-\mathrm{kDa}$ fragment, as observed upon proteolysis of membrane-inserted OmpA (16) (Figure 1D). Similarly, trypsin digestion of A8-35refolded FomA led to a fragment migrating at $34 \mathrm{kDa}$ (in the absence of heat denaturation; Figure 1E), as observed with the native membrane-integrated protein (see ref 32 ). Taken together, biochemical observations are consistent with the two proteins having recovered their native structure and being kept water-soluble thanks to a layer of APol adsorbed onto the outer surface of the $\beta$-barrel.

The functionality of refolded OmpA and FomA was examined by single-channel recordings. Neither protein forms channels when applied to bilayers of diphytanoylphosphatidylcholine (di $\mathrm{PhPC}$ ) in its unfolded form (Figure 2, traces Aa5 and Ba5). After refolding in A8-35 and being transferred to a black film, on the other hand, OmpA formed small, 4-16 pS channels, as well as channels of larger, 250-320 $\mathrm{pS}$ conductance (Figure 2Aa, traces 1 and 2) in $1 \mathrm{M} \mathrm{KCl}$ at $\mathrm{pH}$ 7.2. States with small $(50 \mathrm{pS})$ and large $(260-320 \mathrm{pS})$ conductance have been reported for OmpA refolded in detergent (25). The 4-16 pS channel of A8-35-refolded OmpA was converted to a $70-80 \mathrm{pS}$ channel when the same concentration of A8-35 (without OmpA) was added to the trans compartment of the experimental chamber (Figure 2Aa, trace 3, and Figure 2Ac). The perturbing effect of an asymmetrical distribution of A8-35 is likely related to the fact that APols bind to protein-free lipid bilayers (38), which may affect electrostatic and/or internal pressure gradients.
Similarly, FomA refolded in A8-35 and reconstituted into diPhPC black films featured a single-channel conductance of $0.4 \mathrm{nS}$, versus a value of $1.1 \mathrm{nS}$ for FomA refolded in LDAO ( $N$-lauryl- $N, N$-dimethylammonium $N$-oxide) (Figure 2B). Again, this difference disappeared upon addition of A835 in trans. The two $\beta$-barrel proteins therefore appear fully functional after refolding in APols and being transferred to a lipid bilayer. The ability of A8-35-associated OmpA and FomA to integrate into preformed black films is consistent with previous observations showing that an APol-trapped membrane enzyme, diacylglycerolkinase, can spontaneously partition into lipid vesicles, where it is functional (39).

Refolding an $\alpha$-Helical Membrane Protein, Bacteriorhodopsin. MPs with either a $\beta$-barrel or an $\alpha$-helical transmembrane structure fold according to widely different principles. Renaturation experiments therefore were undertaken using bacteriorhodopsin (BR), a paradigmatic $\alpha$-helical MP. BR, whose transmembrane domain is made up of a bundle of seven $\alpha$-helices (see ref 40 and references therein), accumulates in the plasma membrane of $H$. salinarium, forming the so-called purple membrane (PM) patches, where it is the sole protein. Thanks to a covalently but loosely bound cofactor, retinal, BR functions as a light-driven proton pump (18). When native BR is transferred from an octyl thioglucoside (OTG) solution to A8-35, its absorption spectrum is not affected, including the blue shift induced by solubilization, and it becomes markedly more stable $(7,41)$. Denatured BR releases its cofactor, whose main absorption peak shifts from $\sim 555 \mathrm{~nm}$ (dark-adapted BR) to $382 \mathrm{~nm}$ (free retinal). BR was the first $\alpha$-helical MP ever to be 
renatured, after complete unfolding in organic solvents and transfer to SDS, using as the refolding medium either mixed lipid/detergent micelles (19) or lipid vesicles (20), which established that its native state lies at a free energy minimum (19). Upon renaturation, retinal spontaneously rebinds to the refolded apoprotein, regenerating the characteristic purple color of the holoprotein.

In a first set of experiments, PM was solubilized in SDS, yielding a mixture of PM lipids, bacterioopsin (BO), and retinal in a $1 / 1 \mathrm{BO} /$ retinal molar ratio. This solution, whose near-UV absorbance spectrum is identical to that of free retinal (Figure 3A), reflecting the absence of native BR, was supplemented with A8-35 in various mass ratios, and dodecyl sulfate precipitated as its potassium salt (KDS) (20). The purple color of BR started to develop within minutes. After centrifugation of KDS crystals and removal of residual dodecyl sulfate by dialysis, UV-visible absorption spectra indicated that $\mathrm{BR}$ renaturation was partial at a 2/1 A8-35/ $\mathrm{BO}$ mass ratio and complete at mass ratios of either $5 / 1$ or 10/1 (Figure 3A). When size exclusion chromatography was conducted, the refolded BR migrated mostly as monomeric $\mathrm{BR} / \mathrm{APol}$ particles at the latter two ratios, while aggregation was conspicuous at the lowest one (Figure 3B).

An important theoretical and practical issue when attempting to refold MPs is the extent to which their native environment must be mimicked, in particular by including lipids in an attempt to emulate the physical and chemical properties of a bilayer. Refolding of BR was therefore attempted starting from delipidated BO (dBO): PM was denatured in formic acid (19), the solution supplemented with ethanol, and the apoprotein separated from lipids and retinal by size exclusion chromatography. Unfolded $\mathrm{dBO}$ in a formic acid/ethanol mixture was supplemented with solid SDS and transferred to an aqueous SDS solution by dialysis (20). The absence of PM lipids, which was checked by thin layer chromatography (see Experimental Procedures), is consistent with the complete absence of a retinal absorbance peak in the UV spectra of the $\mathrm{dBO}$ fractions (Figure 3C). After supplementing the solution with retinal and A8-35, renaturation was carried out as for SDS-solubilized PM. UV-visible spectra indicated a high degree of refolding (60-80\%) (Figure 3C). A8-35-refolded, lipid-free BR was stable for at least three weeks at $4{ }^{\circ} \mathrm{C}$, giving no spectral indication of any denaturation. Control experiments carried out under comparable conditions using detergents instead of APols as the acceptor medium showed lower levels of renaturation, ranging from negligible (octyl glucoside, OTG, $\mathrm{C}_{8} \mathrm{E}_{4}$ ) to mediocre $(\sim 1 / 3$ in Triton X-100) to fairly $\operatorname{good}(\sim 2 / 3$ in dodecyl maltoside) (not shown). Renaturation by transfer to A8-35 was also observed using other methods for displacing SDS, such as dilution into an excess of APol or dialysis of SDS from a SDS/APol solution (not shown).

Upon illumination with white light, A8-35-refolded BR underwent the characteristic red shift that indicates that the photocycle is active and populates the light-adapted state (not shown). The functionality of APol-refolded, lipid-free BR (rdBR/APol) was further explored by monitoring the photocycle induced by exciting the light-adapted protein at 640 nm with a 5-ns laser flash. In the explored spectral range (370-500 nm), transient absorption changes, followed over a $10 \mathrm{~ns}$ to $100 \mathrm{~ms}$ time scale, were virtually identical to those observed with A8-35-trapped, lipid-associated native BR
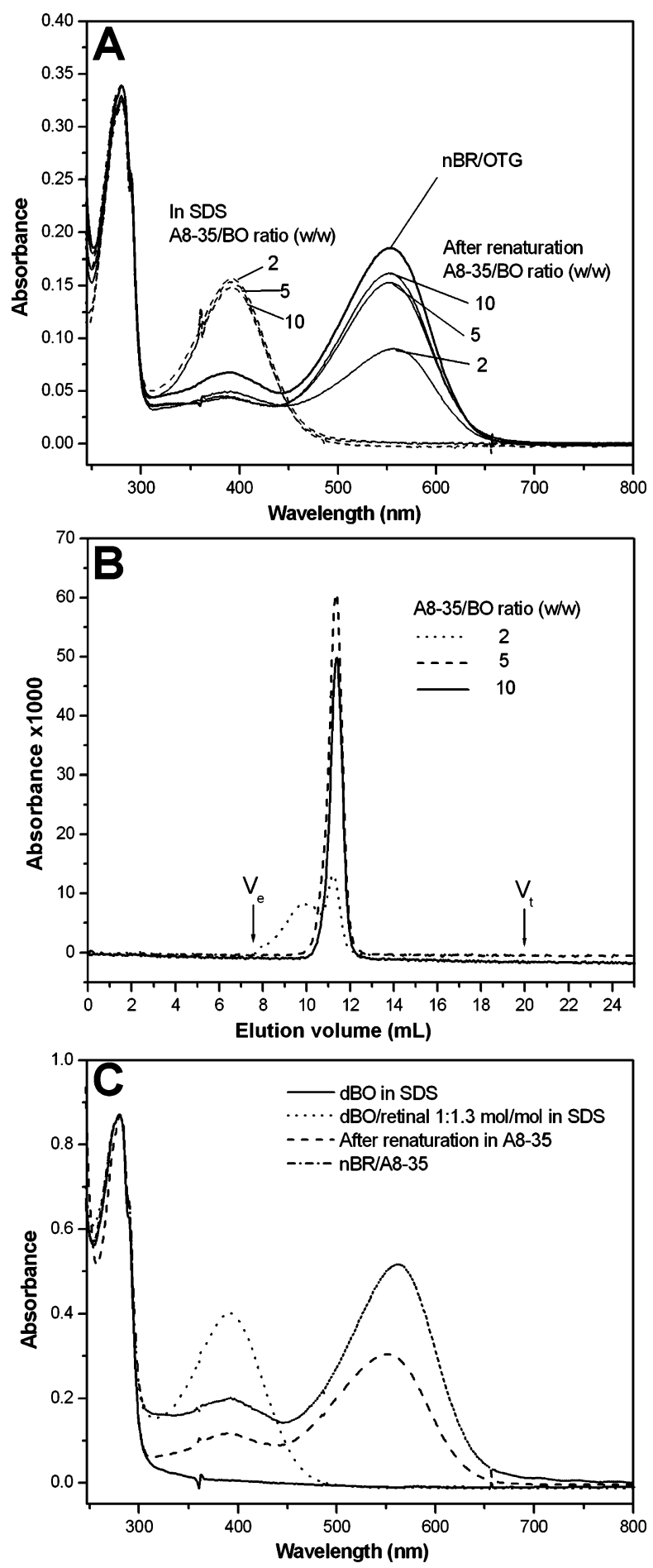

FIGURE 3: Renaturation of bacteriorhodopsin in A8-35. (A) Renaturation from SDS-solubilized purple membrane. Absorbance spectra of PM supplemented with A8-35 at various weight ratios, in SDS solution (- - -) and after precipitation and dialysis of dodecyl sulfate (-). An absorbance spectrum of native BR in OTG is shown for comparison (nBR/OTG). (B) Size exclusion chromatography analysis of the refolded samples from panel $\mathrm{A}$, analyzed at either $554 \mathrm{~nm}\left(2 / 1\right.$ and 10/1 A8-35/BO ratios) or $570 \mathrm{~nm}$ (5/1 ratio). $V_{\mathrm{e}}$ is the excluded volume and $V_{\mathrm{t}}$ the total volume. (C) Renaturation of purified, delipidated $\mathrm{BO}(\mathrm{dBO})$. Absorbance spectra of $\mathrm{dBO}$ in SDS solution $(-)$, after addition of retinal $(\cdots)$, and after renaturation (- - ). The spectrum of native BR trapped in A8-35 is shown for comparison $(-\cdot)$. Spectra have been normalized to the same value of $A_{280}$.

(nBR/APol), which themselves are similar, although not identical, to those observed in a detergent solution (nBR/ 


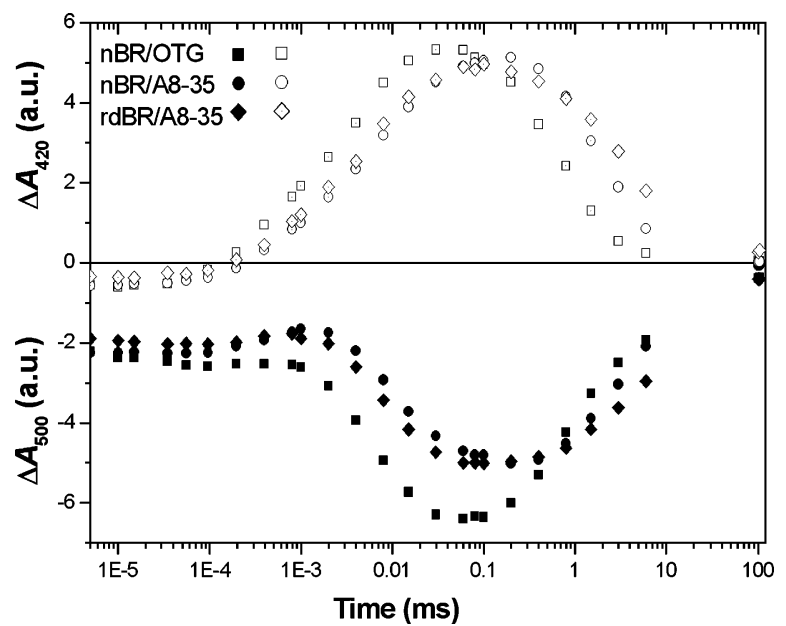

FIGURE 4: Photocycle of native, A8-35-trapped BR in Na buffer [nBR/APol $(\mathrm{O}$ and $\bullet)]$ and of BR refolded in A8-35 from dBO and retinal in $\mathrm{K}$ buffer [rdBR/APol $(\diamond$ and $\diamond)$ ], followed at 420 $\mathrm{nm}$ (empty symbols) and $500 \mathrm{~nm}$ (filled symbols) after a $5 \mathrm{~ns}$ actinic light pulse. Data obtained with native BR in OTG solution in $\mathrm{Na}$ buffer [nBR/OTG ( $\square$ and $\mathbf{\square}$ )] are shown for comparison. At the wavelengths that were used, absorbance changes reflect primarily although not purely the rise and decay of the M states.

OTG) (Figure 4). A discussion of the nature and origin of differences between photocycle kinetics of BR in either a detergent or an APol environment is beyond the scope of this work and will be presented elsewhere.

\section{DISCUSSION}

The observations described in the present article, are of both basic and practical interest. From a theoretical point of view, it is remarkable that several structurally highly different MPs could be refolded in a medium so unlike their native membrane environment. When solutions of denatured MPs are depleted of denaturant, in our examples, urea or SDS, in the presence of APols, refolding proteins must find themselves entangled with an amphipathic polymer whose physical and chemical properties bear little similarity with those of a membrane environment. Nevertheless, they reach their native fold with high efficiency, and this even in the complete absence of lipids. This strongly suggests that, at least for the particular set of MPs studied here, the detailed chemical composition and physical properties of the environment are not essential in determining the conformation of the polypeptide chain that features the lowest standard free energy: the availability of a nondenaturing amphipathic screen that shields the transmembrane region from water suffices, the rest of the physical and chemical information needed to achieve the native 3D structure being provided by the amino acid sequence. This is consistent with the view that detergents interfere with refolding, and are inactivating, more because of their dissociating properties (they compete with stabilizing protein-protein and protein-lipid interactions) than because they do not provide bilayer-like constraints (cf. ref 42). It is well-established that their membrane environment can affect the structure, stability, and function of MPs. The data presented here suggest that, at least for the three proteins studied, the amino acid sequence nevertheless encodes sufficiently strong constraints for the native state to be reached in an environment that mimics, at best, only very crudely that in which the protein naturally inserts in vivo.

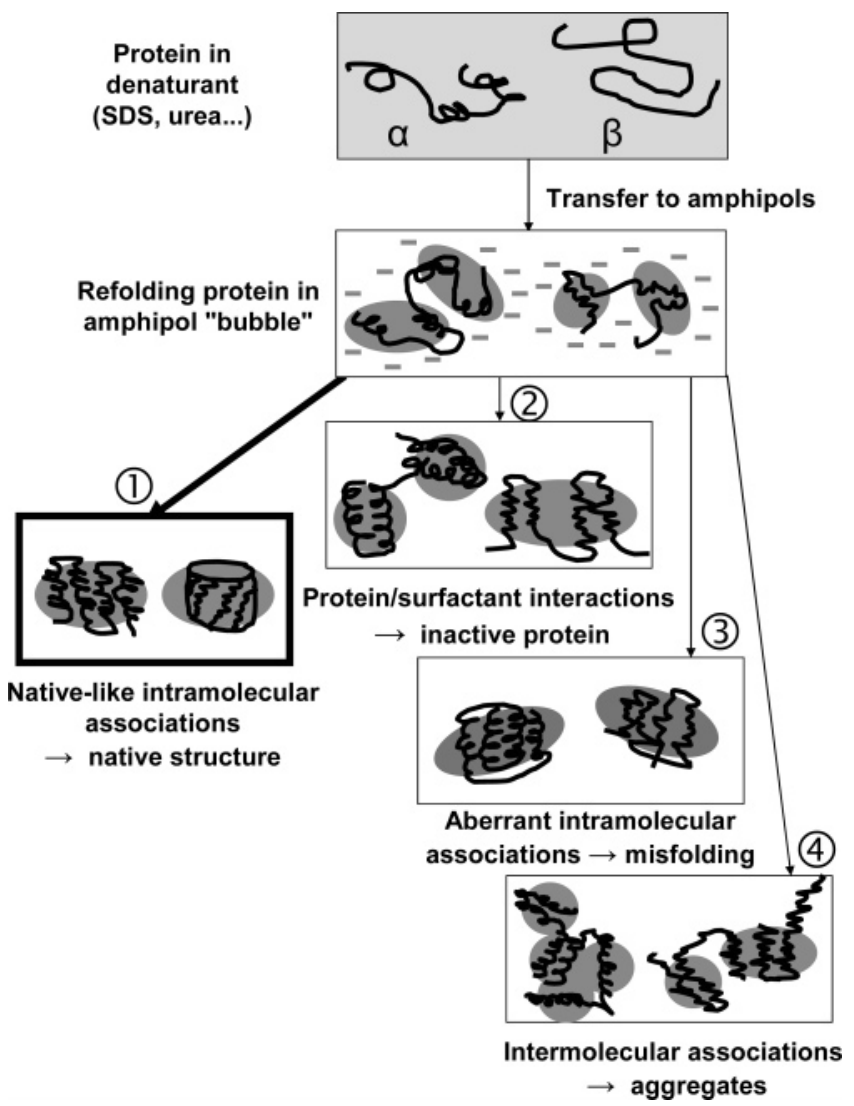

FIGURE 5: Hypothetical scheme of events leading to either refolding to the native state (1)), incomplete refolding (2), misfolding (3), or aggregation (4) upon removal of the denaturant from a solution of the denatured membrane protein. A8-35 is postulated to favor pathway (1). See the text.

BO preserves (or, after being transferred from organic solvents, recovers) some secondary structure in SDS (19) and likely exposes to its surroundings $\alpha$-helical hydrophobic surfaces. Since it is known that, in the simultaneous presence of detergent and APols, mixed surfactant layers surround native MPs (see, e.g., refs 8,12 , and 43), it is possible that, upon addition of A8-35, partially folded helices become surrounded by a mixture of APols and SDS. Denatured OmpA and FomA, on the other hand, do not exhibit any hydrophobic surfaces whether in urea solutions or water $(16$, $34)$. In our experiments, where denatured $\beta$-barrel proteins simultaneously experience urea dilution and exposure to APols, the hydrophobic surface of the barrel does not exist prior to the transfer. APol-mediated refolding of the two types of MPs therefore may well involve quite different steps and transient modes of association with APols before the native structure is reached.

Why APols, which are perfectly foreign molecules, unlike any of those with which a MP normally comes into contact with in a cell, provide a favorable medium for refolding will deserve a detailed investigation. We can, at this stage, provide only tentative suggestions (Figure 5). We assume that, upon removal of the denaturant, some sequence regions start to refold, and specific interactions between protein secondary structure elements reappear. Those can be intramolecular, which can lead either to renaturation, if they are native-like (1), or, if they are not, to misfolding (3), or intermolecular, which creates aggregation (4). Protein-surfactant interactions, competing with protein-protein ones, may prevent 
their re-formation (2)). One of the key difficulties in refolding MPs is to identify conditions that favor path (1) over the others and/or permit recovery from non-native states. One way to avoid aggregation is to immobilize MPs to be refolded onto a solid matrix, preventing intermolecular interactions (44-46). In solution, on the other hand, a delicate balance must be reached between keeping the refolding proteins apart, in preventing aggregation, and hampering renaturation by overly dissociating an environment. The rationale behind the efficiency of A8-35 at promoting refolding is, very likely, that it has enough affinity for MPs to prevent or slow intermolecular associations between refolding proteins, while being unable to block the (re)formation of those intramolecular interactions that determine and stabilize their 3D structure (Figure 5). This may be related to the fact that the multipoint attachment characteristic of APols can provide high-affinity binding even in the absence of strong local interactions. A8-35, in this hypothesis, could be considered as enclosing refolding MPs in a sort of protective, nondenaturing "bubble", much in the same manner as chaperone proteins like GroE facilitate in vivo folding of soluble proteins (47). The same mechanism may well contribute to the protection of APol-trapped native MPs against denaturation.

The fact that A8-35 provides an efficient refolding medium for one $\alpha$-helical and two $\beta$-barrel MPs suggests that this procedure could possibly be extended to other MPs. Given the considerable basic and biomedical importance of obtaining large amounts of properly folded MPs for structural and pharmacological studies and the problems presented by largescale expression of most MPs in their native form, APols may represent an attractive alternative tool for folding them from inclusion bodies. Once folded in APols, MPs will not necessarily need to be exposed to detergents, since many functional or structural studies, including ligand binding measurements (13) and solution NMR investigations (15), can be carried out directly on MP-APol complexes. Alternatively, APols, once renaturation is over, can easily be replaced either with detergents $(31,43)$ or with lipids. The latter exchange can be achieved either directly, by exposing MP-APol complexes to a preformed membrane (ref 39 and this work), or indirectly, via a detergentassociated intermediate that is then subjected to conventional reconstitution.

\section{ACKNOWLEDGMENT}

We are grateful to H. Jensen (University of Bergen) for providing the FomA plasmid, to F. Giusti (UMR 7099) for the synthesis of A8-35 and for his help with lipid analyses, to F. Lebaupain (UMR 7099) for her participation in the preparation of $\mathrm{dBO}$, and to M. E. Dumont (University of Rochester, Rochester, NY) and P. Champeil (CEA, Saclay, France) for reading an earlier version of the manuscript. P.-L. Porté deserves special thanks for his help with graphics.

\section{REFERENCES}

1. Tate, C. G. (2001) Overexpression of mammalian integral membrane proteins for structural studies, FEBS Lett. 504, 9498.

2. Drew, D., Froderberg, L., Baars, L., and De Gier, J. W. (2003) Assembly and overexpression of membrane proteins in Escherichia coli, Biochim. Biophys. Acta 1610, 3-10.
3. Buchanan, S. K. (1999) $\beta$-Barrel proteins from bacterial outer membranes: Structure, function and refolding, Curr. Opin. Struct. Biol. 9, 455-461.

4. Kiefer, H. (2003) In vitro folding of $\alpha$-helical membrane proteins, Biochim. Biophys. Acta 1610, 57-62.

5. Booth, P. J. (2003) The trials and tribulations of membrane protein folding in vitro, Biochim. Biophys. Acta 1610, 51-56.

6. Kleinschmidt, J. H. (2005) Folding and stability of monomeric $\beta$-barrel membrane proteins, in Protein-lipid interactions: From membrane domains to cellular networks (Tamm, L. K., Ed.) pp 111-140, Wiley-VCH, Weinheim, Germany.

7. Tribet, C., Audebert, R., and Popot, J.-L. (1996) Amphipols: Polymers that keep membrane proteins soluble in aqueous solutions, Proc. Natl. Acad. Sci. U.S.A. 93, 15047-15050.

8. Popot, J.-L., Berry, E. A., Charvolin, D., Creuzenet, C., Ebel, C., Engelman, D. M., Flötenmeyer, M., Giusti, F., Gohon, Y., Hervé, P., Hong, Q., Lakey, J. H., Leonard, K., Shuman, H. A., Timmins, P., Warschawski, D. E., Zito, F., Zoonens, M., Pucci, B., and Tribet, C. (2003) Amphipols: Polymeric surfactants for membrane biology research, Cell. Mol. Life Sci. 60, 1559-1574.

9. Picard, M., Dahmane, T., Garrigos, M., Gauron, C., Giusti, F., le Maire, M., Popot, J.-L., and Champeil, P. (2006) Protective and inhibitory effects of various types of amphipols on the $\mathrm{Ca}^{2+}$ ATPase from sarcoplasmic reticulum: A comparative study, Biochemistry 45, 1861-1869.

10. Gohon, Y., Pavlov, G., Timmins, P., Tribet, C., Popot, J.-L., and Ebel, C. (2004) Partial specific volume and solvent interactions of amphipol A8-35, Anal. Biochem. 334, 318-334.

11. Gohon, Y., Giusti, F., Prata, C., Charvolin, D., Timmins, P., Ebel, C., Tribet, C., and Popot, J.-L. (2006) Well-defined nanoparticles formed by hydrophobic assembly of a short and polydisperse random terpolymer, amphipol A8-35, Langmuir 22, 1281-1290.

12. Champeil, P., Menguy, T., Tribet, C., Popot, J.-L., and le Maire, M. (2000) Interaction of amphipols with the sarcoplasmic $\mathrm{Ca}^{2+}$ ATPase, J. Biol. Chem. 275, 18623-18637.

13. Martinez, K. L., Gohon, Y., Corringer, P.-J., Tribet, C., Mérola, F., Changeux, J.-P., and Popot, J.-L. (2002) Allosteric transitions of Torpedo acetylcholine receptor in lipids, detergent and amphipols: Molecular interactions vs. physical constraints, FEBS Lett. 528, 251-256.

14. Sanders, C. R., Hoffmann, A. K., Gray, D. N., Keyes, M. H., and Ellis, C. D. (2004) French swimwear for membrane proteins, ChemBioChem 5, 423-426.

15. Zoonens, M., Catoire, L. J., Giusti, F., and Popot, J.-L. (2005) NMR study of a membrane protein in detergent-free aqueous solution, Proc. Natl. Acad. Sci. U.S.A. 102, 8893-8898.

16. Surrey, T., and Jähnig, F. (1992) Refolding and oriented insertion of a membrane protein into a lipid bilayer, Proc. Natl. Acad. Sci. U.S.A. 89, 7457-7461.

17. Qi, S. Y., Li, Y., and O' Connor, C. D. (1994) The region around residue 115 of human bactericidal/permeability-increasing protein is not involved in lipopolysaccharide binding or bactericidal activity. Chemical synthesis and expression of a gene coding for the active domain and characterization of recombinant proteins, Biochem. J. 298, 711-718.

18. Oesterhelt, D., and Stoeckenius, W. (1971) Rhodopsin-like protein from the purple membrane of Halobacterium salinarium, Nat. New Biol. 233, 149-152.

19. Huang, K.-S., Bayley, H., Liao, M.-J., London, E., and Khorana, H. G. (1981) Refolding of an integral membrane protein. Denaturation, renaturation, and reconstitution of intact bacteriorhodopsin and two proteolytic fragments, J. Biol. Chem. 256, 3802-3809.

20. Popot, J.-L., Gerchman, S.-E., and Engelman, D. M. (1987) Refolding of bacteriorhodopsin in lipid bilayers: A thermodynamically controlled two-stage process, J. Mol. Biol. 198, 655676.

21. Corcelli, A., Colella, M., Mascolo, G., Fanizzi, F. P., and Kates, M. (2000) A novel glycolipid and phospholipid in the purple membrane, Biochemistry 39, 3318-3326.

22. Liao, M.-J., London, E., and Khorana, H. G. (1983) Regeneration of the native bacteriorhodopsin structure from two chymotryptic fragments, J. Biol. Chem. 258, 9949-9955.

23. Rehorek, M., and Heyn, M. P. (1979) Binding of all-trans-retinal to the purple membrane. Evidence for cooperativity and determination of the extinction coefficient, Biochemistry 18, 49774983. 
24. Benz, R., Janko, K., Boos, W., and Läuger, P. (1978) Formation of large, ion-permeable membrane channels by the matrix protein (porin) of Escherichia coli, Biochim. Biophys. Acta 511, 305319.

25. Arora, A., Rinehart, D., Szabo, G., and Tamm, L. K. (2000) Refolded outer membrane protein A of Escherichia coli forms ion channels with two conductance states in planar lipid bilayers, J. Biol. Chem. 275, 1594-1600.

26. Kleivdal, H., Benz, R., and Jensen, H. B. (1995) The Fusobacterium nucleatum major outer-membrane protein (FomA) forms trimeric, water-filled channels in lipid bilayer membranes, Eur. J. Biochem. 233, 310-316.

27. Béal, D., Rappaport, F., and Joliot, P. (1999) A new highsensitivity 10 -ns time-resolution spectrophotometric technique adapted to in vivo analysis of the photosynthetic apparatus, Rev. Sci. Instrum. 70, 202-207.

28. Pautsch, A., and Schulz, G. E. (2000) High-resolution structure of the OmpA membrane domain, J. Mol. Biol. 298, 273-282.

29. Sonntag, I., Schwarz, H., Hirota, Y., and Henning, U. (1978) Cell envelope and shape of Escherichia coli: Multiple mutants missing the outer membrane lipoprotein and other major outer membrane proteins, J. Bacteriol. 136, 280-285.

30. Rawling, E. G., Brinkman, F. S., and Hancock, R. E. (1998) Roles of the carboxy-terminal half of Pseudomonas aeruginosa major outer membrane protein $\mathrm{OprF}$ in cell shape, growth in lowosmolarity medium, and peptidoglycan association, J. Bacteriol. $180,3556-3562$.

31. Zoonens, M. (2004) Caractérisation des complexes formés entre le domaine transmembranaire de la protéine OmpA et des polymères amphiphiles, les amphipols. Application à l'étude structurale des protéines membranaires par RMN à haute résolution, Doctorat d'Université, Université Paris-6, Paris.

32. Puntervoll, P., Ruud, M., Bruseth, L. J., Kleivdal, H., Hogh, B. T., Benz, R., and Jensen, H. B. (2002) Structural characterization of the fusobacterial non-specific porin FomA suggests a 14stranded topology, unlike the classical porins, Microbiology 148, 3395-3403.

33. Pocanschi, C. L., Apell, H.-J., Puntervoll, P., Høgh, B. T., Jensen, H. B., Welte, W., and Kleinschmidt, J. H. (2005) The major outer membrane protein of Fusobacterium nucleatum (FomA) folds and inserts into lipid bilayers via parallel folding pathways, J. Mol. Biol. 355, 548-561

34. Surrey, T., and Jähnig, F. (1995) Kinetics of folding and membrane insertion of a $\beta$-barrel membrane protein, J. Biol. Chem. 270, 28199-28203.

35. Sugawara, E., Steiert, M., Rouhani, S., and Nikaido, H. (1996) Secondary structure of the outer membrane proteins OmpA of Escherichia coli and OprF of Pseudomonas aeruginosa, J. Bacteriol. 178, 6067-6069.
36. Schweizer, M., Hindennach, I., Garten, W., and Henning, U. (1978) Major proteins of the Escherichia coli outer cell envelope membrane. Interaction of protein II with lipopolysaccharide, Eur. J. Biochem. 82, 211-217.

37. Bakken, V., Aaro, S., and Jensen, H. B. (1989) Purification and partial characterization of a major outer-membrane protein of Fusobacterium nucleatum, J. Gen. Microbiol. 135, 3253-3262.

38. Ladavière, C., Toustou, M., Gulik-Krzywicki, T., and Tribet, C. (2001) Slow reorganization of small phosphatidylcholine vesicles upon adsorption of amphiphilic polymers J. Colloid Interface Sci. $241,178-187$

39. Nagy, J. K., Kuhn Hoffmann, A., Keyes, M. H., Gray, D. N., Oxenoid, K., and Sanders, C. R. (2001) Use of amphipathic polymers to deliver a membrane protein to lipid bilayers FEBS Lett. 501, 115-120.

40. Neutze, R., Pebay-Peyroula, E., Edman, K., Royant, A., Navarro, J., and Landau, E. M. (2002) Bacteriorhodopsin: A high-resolution structural view of vectorial proton transport, Biochim. Biophys. Acta 1565, 144-167.

41. Gohon, Y. (2002) Etude structurale et fonctionnelle de deux protéines membranaires, la bactériorhodopsine et le récepteur nicotinique de l'acétylcholine, maintenues en solution aqueuse non détergente par des polymères amphiphiles, Thèse de Doctorat d'Université, Université Paris-VI, Paris.

42. Gohon, Y., and Popot, J.-L. (2003) Membrane protein-surfactant complexes, Curr. Opin. Colloid Interface Sci. 8, 15-22.

43. Tribet, C., Audebert, R., and Popot, J.-L. (1997) Stabilization of hydrophobic colloidal dispersions in water with amphiphilic polymers: Application to integral membrane proteins, Langmuir 13, 5570-5576.

44. Banères, J.-L., Martin, A., Hullot, P., Girard, J.-P., Rossi, J.-C., and Parello, J. (2003) Structure-based analysis of GPCR function. Conformational adaptation of both agonist and receptor upon leukotriene $\mathrm{B}_{4}$ binding to recombinant BLT1, J. Mol. Biol. 329, 801-814.

45. Banères, J.-L., Mesnier, D., Martin, A., Joubert, L., Dumuis, A., and Bockaert, J. (2005) Molecular characterization of a purified $5-\mathrm{HT}_{4}$ receptor. A structural basis for drug efficacy, J. Biol. Chem 280, 20253-20260.

46. Mogensen, J. E., Tapadar, D., Schmidt, M. A., and Otzen, D. E. (2005) Barriers to folding of the transmembrane domain of the Escherichia coli autotransporter adhesin involved in diffuse adherence, Biochemistry 44, 4533-4545.

47. Walter, S. (2002) Structure and function of the GroE chaperone Cell. Mol. Life Sci. 59, 1589-1597.

BI0616706 